
\title{
Reseacch Soute \\ Resilience To Suicidal Behaviour In Young Adults: A Cross-Sectional Study
}

\author{
Jin Han ( $\boldsymbol{\nabla}$ j.han@blackdog.org.au ) \\ University of New South Wales \\ lana Wong \\ University of New South Wales \\ Helen Christensen \\ University of New South Wales \\ Philip J Batterham \\ The Australian National University
}

\section{Research Article}

Keywords: Resilience, suicide, suicidal behaviour, young people

Posted Date: February 9th, 2022

DOI: https://doi.org/10.21203/rs.3.rs-1233434/v1

License: (9) This work is licensed under a Creative Commons Attribution 4.0 International License. Read Full License 


\section{Abstract}

Despite decades of research on suicide risk factors in young people, there has been no significant improvement in our understanding of this phenomenon. This study adopts a positive deviance approach to identify the individuals with suicide resilience and to describe their associated psychological and sociodemographic profiles. Australian young adults aged 18 to 25 years with suicidal thoughts $(N=557)$ completed an online survey covering sociodemographic, mental health status, emotion regulatory and suicide-related domains. Latent class analysis was used to identify the individuals with suicide resilience. Predictors of suicide resilience were assessed using logistic regression models. Results suggested that one in ten $(n=55)$ met criteria for suicide resilience. Factors that had a significant association with suicide resilience included greater cognitive flexibility, greater self-efficacy in expressing positive affect, reduced use of digital technology and less self-harm and substance use as a response to emotional distress. This study identified the factors that may protect young adults with suicidal thoughts from progressing to suicide attempts. Suicide prevention programs might be optimised by shifting from a deficit-based to a strength-based approach through promoting cognitive flexibility, self-efficacy and reducing maladaptive coping.

\section{Introduction}

Suicide is a complex public health phenomenon that represents a leading cause of death for young adults aged between 18 and 25 years globally and in Australia ${ }^{1}$. Research in this field has mostly focused on detecting the risk factors of suicide with a noticeable scarcity of studies on suicide resilience 2-4. Shifting our attention to resilience to suicide and its related behaviour may help inform innovative suicide prevention strategies ${ }^{5,6}$. Developing strengths-based approaches can be an important new direction in developing interventions, as a focus on risks of suicide has not advanced our understanding of suicide or reduce suicide rates ${ }^{7}$.

Suicide resilience is generally acknowledged as both the ability and the dynamic process of maintaining psychological and physical health functioning under high levels of suicide risks ${ }^{8,9}$. The concept is largely derived from well-documented observations that the majority of high-risk individuals, e.g., those with depression ${ }^{10}$ or suicidal thoughts ${ }^{11}$, do not develop suicidal behaviour. Most of the current studies on suicide resilience adopt the buffering hypothesis ${ }^{8}$ to identify the psychological constructs that moderate the relationship between suicide risks and suicidal behaviour ${ }^{12,13}$. This approach, however, is often limited because it yields relatively small effect sizes (2\%-5\%) of the indicated interaction effects and inconsistent findings across studies, while a person-centered approach by identifying resilient individuals demonstrates stability of resilience over time ${ }^{14}$.

Positive deviance (PD) is a person-centered research approach that is promising to disentangle the relationship between suicide risks and suicidal behaviour. This approach is gaining prominence in identifying and understanding individuals' exceptional performance under complex health situations ${ }^{15-}$ 
17. The PD approach is grounded on the premise that in each community there are certain individuals whose uncommon behaviour and strategies enable them to find better solutions to the same problems than their peers ${ }^{18}$. It has been effectively implemented as a practical strategy to untangle complex health problems, such as child malnutrition ${ }^{19}$, safe sexual practices ${ }^{20}$, and weight control ${ }^{21}$. To our knowledge, the PD approach has not been implemented in suicide prevention.

In this study, we followed the general practice in PD research to firstly define the problem ${ }^{22}$. We aim to understand the factors that promote resilience to suicidal behaviour amongst young people with suicidal thoughts, as the transition from suicidal thoughts to behaviour is increasingly acknowledged as a significant predictor of suicide death in theories ${ }^{23,24}$ and empirical literature ${ }^{23,24}$.

One area that warrants further investigation in the suicidal ideation to action framework is emotion regulation ${ }^{25}$. Although extensive research has indicated that emotion dysregulation is associated with suicidal thoughts and history of suicidal attempts after accounting for the symptoms of psychological disorders ${ }^{26-29}$, the relationship between emotion dysregulation and suicidal behaviour is complex.

On one hand, there is evidence that emotion dysregulation is closely related to increased risks of engaging in risky behaviour ${ }^{30}$, such as deliberate self-harm ${ }^{31}$ and alcohol use ${ }^{32}$. These associations may be mediated by increased acquired capability for suicide, a proposed key factor in the transition from suicidal thoughts to attempts ${ }^{33}$. On the other hand, deficits in emotion regulation also indicate individuals' low ability to tolerate distress, as behaviour of emotionally dysregulated individuals is often motivated by their desire to escape from painful experiences ${ }^{34}$. Emotion dysregulation can thus serve to counter or lower acquired capability for suicide ${ }^{35}$. The inconsistent findings may be related to different aspects of emotion regulation, such as the adoption of maladaptive emotion regulation strategies such as self-harm behaviour ${ }^{36}$, ability to switch between emotional regulation strategies in adaption to situations (flexibility) ${ }^{37,38}$, or confidence in the ability to cope with different stressors (self-efficacy) ${ }^{39,40}$. Therefore, further investigation in the context of youth suicide prevention is required to better understand the roles of these related components of emotion regulation.

Therefore, this study seeks to, for the first time, understand resilience to suicidal behaviour amongst young adults with suicidal thoughts via the positive deviance informed analytic approach. We hypothesized that young people with suicide resilience would be distinguished by more adaptive emotion regulation strategies, higher coping and cognitive flexibility and higher self-efficacy in emotion regulation, after adjusting for constraints (i.e., risk factors of suicidal attempts). We expected that our results would improve our understanding of protective factors to optimise suicide prevention interventions and facilitate the transition from a deficit-based to a strength-based suicide prevention approach. Identifying psychological, social and behavioural profiles of young people who demonstrate positive deviance may indicate specific therapeutic targets, thereby guiding the development of more effective treatment and prevention interventions for individuals with suicidal thoughts. 


\section{Method}

\subsection{Participants and procedure}

Australian young adults aged between 18 and 25 years who experienced suicidal thoughts in the past year were recruited from Facebook and Instagram advertisements in February 2021. Other eligibility criteria include being fluent in English, currently living in Australia, having no diagnosis of bipolar disorder or psychosis, and having no suicide attempt in the past 30 days. Informed consent was obtained from all participants. Eligible participants then filled in a Qualtrics online survey, including questions on sociodemographic (e.g., age, sex, socioeconomic status), mental and physical health conditions and status (e.g., suicidal thoughts and behaviour, depression, anxiety), emotion regulation and related constructs (e.g., coping flexibility, cognitive flexibility, regulatory emotional self-efficacy), and health service usage (e.g., help-seeking intentions). The participants were reimbursed for their participation by a draw to win one of three $\$ 30$ e-gift vouchers. From the 2392 clicks on the Facebook and the Instagram advertisements, 725 participants completed the eligibility assessment, of which 658 (90.8\%) were eligible, and $557(84.7 \%)$ completed the variables of interest for the current study. No significant difference was found between the complete and incomplete responses on age and sex. The research was carried out in accordance with the latest version of the Declaration of Helsinki, and the study received ethics approval from the Human Research Ethics Committee at the University of New South Wales (HC200696).

\subsection{Measures}

Sociodemographic variables include date of birth, sex, sexual orientation, current living and relationship status, highest level of education completed, self-perceived socioeconomic status ${ }^{41}$, history of diagnosed mental illness and long-term physical health conditions, and current medication.

Mental and physical wellbeing measures include subjective mental wellbeing measured by the Short Warwick-Edinburgh Mental Well-Being Scale SWEMWBS; ${ }^{42}$. The scale consists of seven items assessing general mental wellbeing over the past two weeks. Total converted scores range from 7 to 35 with higher scores indicating higher levels of subjective mental well-being. This scale has demonstrated good reliability in the current study (Cronbach's $\mathrm{a}=0.82$ ). Physical wellbeing was measured by the EQ-VAS ${ }^{43}$, a visual analogue scale numbered from 0 (the worst health you can imagine) to 100 (the best health you can imagine) over the last two weeks. A higher score represents a perception of better physical health.

The severity of suicidal thoughts was assessed by the Suicidal Ideation Attributes Scale SIDAS; ${ }^{44}$, consisting of five items related to frequency of suicidal thoughts, controllability, closeness to suicidal attempt, levels of distress and impact on daily functioning in the past month. Item two (controllability) is reverse scored. Total scale ranges from 0 to 50 , with higher scores indicating more severe suicidal thoughts. The measure has shown internal consistency (Cronbach's $a=0.84$ ) in the current study. History of suicide attempts was assessed by a question on a three-point Likert scale, ranging from "No, never (0)", "Yes, once (1)", to "Yes, more than once (2)" 45. 
Levels of depression and anxiety over the past two weeks were measured by the Patient Health Questionnaire-9 PHQ-9; ${ }^{46}$ and the Generalized Anxiety Disorder-7 GAD-7; 47 , respectively, with higher scores reflecting more severe depression and anxiety. Both measures have shown good internal consistency (PHQ-9, Cronbach's $a=0.86)$ and (GAD-7, Cronbach's $a=0.88)$ in this study. The positive and negative affect was assessed by the International Positive and Negative Affect Schedule Short-form IPANAS-SF; ${ }^{48}$. The scale has ten items assessing the frequency of positive affect (active, determined, attentive, inspired, and alert) and negative affect (afraid, nervous, upset, hostile, and ashamed) over the last two weeks on 5-point Likert scale, ranging from "Never (1)" to "Always (5)". This scale has shown acceptable consistency (Cronbach's $a=0.72$ for positive affect and Cronbach's $a=0.68$ for negative affect) in the current study.

Emotion regulation related measures include the 16-item Brief Difficulties in Emotion Regulation Scale DERS-16; ${ }^{49}$ evaluating emotion regulation difficulties: emotional clarity, goals, impulsivity, strategies, and non-acceptance. Total score ranges from 16 to 80 , with higher scores reflecting greater levels of emotion dysregulation. The DERS-16 has shown good internal consistency and in the current study (Cronbach's a $=0.91$. .

Levels of flexibility were measured by the Coping Flexibility Scale CFS; ${ }^{50}$ and the Cognitive Flexibility Scale CFS; ${ }^{51}$ respectively. The Coping Flexibility Scale has 10 items, and items 2 and 7 are reverse coded. The total score ranges from 0 to 30 with higher scores indicating higher ability of coping flexibility. The Cognitive Flexibility Scale comprises 12 items measuring aspects of cognitive flexibility relevant to effective interactions and communication. Items $2,3,5$, and 10 items are reverse scored and the total scores of the scale range between 12 and 72 . Higher scores indicate stronger cognitive flexibility. Both scales demonstrated good internal consistency (Coping Flexibility Scale: Cronbach's a $=0.77$, Cognitive Flexibility Scale: Cronbach's $a=0.75)$ in the current study.

Self-efficacy in emotion regulation was measured by the Regulatory Emotional Self-efficacy Scale RESE; 52. The scale is composed of 12 items that assess competence belief in regulating affects due to occurrence of positive or negative events. This scale assesses self-efficacy in three aspects of emotion regulation: expressing positive emotions, managing despondency-distress, and managing anger-irritation. Each aspect is measured by four items, with total scores of each subscale ranging from 4 to 20 . All subscales have shown good internal consistency in this study, with Cronbach's a ranging from 0.71 to 0.80 .

Cognitive and behavioural responses to emotional distress were measured by 18 questions assessing participants' likelihood to engage in the listed activities ${ }^{53,54}$ using a four-point Likert scale, ranging from "Highly unlikely (1)" to "Highly likely (4)". The activities cover six aspects, including digital technology (i.e., watching TV or online videos, browsing social media, playing videogames or computer games), create arts (i.e., jigsaws, drawing or journaling), self-harm and substance use (i.e., using drug or alcohol, self-harm, thinking about death), exercise (i.e., anerobic exercise, aerobic exercise), self-transcendence (i.e., pray, mindfulness or mediation, challenging negative thoughts, doing randomly kind things to 
others), and selfcare (i.e., sleeping, taking a bath or long shower, playing with pets, massaging), with average scores of each aspect ranging from one to four. Higher scores indicate higher intentions to engage with the activities.

\subsection{Statistical analysis}

Latent class analysis (LCA) was used to identify the positive deviant (PD) cases. LCA is a mixture modelling technique that classifies a seemingly heterogeneous sample into a discrete number of subgroups ('classes'), focusing on the similarities and differences across individuals ${ }^{55}$. Whereas LCA is typically seen as a data-driven approach where the best fitting model is chosen based on statistical consideration, theoretical and practical insights can also be used to optimise the model ${ }^{56}$. In this study, the latent class analysis was performed based on three constructs: subjective mental wellbeing measured by the SWEMWBS, physical wellbeing measured by the EQ-VAS, and existence of self-reported lifetime suicidal attempts. These constructs were chosen to identify the PD individuals who maintained high levels of wellbeing and had not attempted suicide to investigate the resilient factors to suicidal behaviour. A 2-class, 3-class, 4-class, and 5-class models were specified via Mplus version 8, including Bayesian Information Criterion (BIC), Adjusted Bayesian Information Criterion (ABIC), significant levels of bootstrapped likelihood ratio test (BLRT), and the percentages of the total sample across class membership. The model was selected based the significant BLRT, the most robust indicator of class membership, and no classes with $<5 \%$ of the total sample ${ }^{57}$.

Subsequent analyses were conducted with IBM SPSS Statistics (Version 26). To compare the bit-fitting classes on sociodemographic, mental and physical health conditions, and emotion regulation related factors variables, independent sample t-tests were conducted for continuous variables, and chi-square tests were conducted for dichotomous variables. The predictors of PD class membership were assessed firstly using base models including each individual predictor. Significant predictors were then included in a multivariate logistic regression model to assess the impact while adjusting for potentially confounding effects. Significance levels were set at $p<0.05$. All analyses were performed using SPSS Version 26 (SPSS Inc, Chicago, IL, USA).

\section{Results}

\subsection{Classification of PD and non-PD cases}

Table 1 displays the fit statistics for the latent class analysis (Table 1a) and the description of latent classes (Table $1 \mathrm{~b}$ ). This study tested models with 2-5 classes, all of which were supported by BLRT except the 5-class model. Whilst ABIC and BIC supported a 5-class model, membership of the fifth class in the model was low, comprising $3.1 \%$ of the total sample. The 3-class and the 4-class models also included a class of less than $5 \%$ of the total sample. Consequently, the 2 -class model was retained in this study. Membership for the 2-class model was as follows: Class 1 had 502 individuals (90.1\% of the total sample) with $53.4 \%$ who reported lifetime suicidal attempts and significantly lower subjective mental 
(17.5 vs 22.4, $t=-13.18, d f=555, p<.001)$ and physical (46.3 vs 79.1, $t=-18.96, d f=100.6, p<.001)$ wellbeing than Class 2 ( $n=55,9.9 \%$ of the total sample). Participants in Class 2 hereafter are referred as positive deviant (PD) cases who demonstrated resilience to suicidal behaviour and high mental and physical wellbeing.

Table 1

a. Fit statistics and class membership for the identification of PD cases $(n=557)$

\section{Class membership}

$\begin{array}{lllllllll}\text { Model } & \text { BIC } & \text { ABIC } & \text { BLRT } & \mathbf{1} & \mathbf{2} & \mathbf{3} & \mathbf{4} & \mathbf{5} \\ \begin{array}{l}\text { 2- } \\ \text { class }\end{array} & 8592.4 & 8567.0 & <.001 & \begin{array}{l}502 \\ (90.1 \%)\end{array} & 55(9.9 \%) & & & \\ \begin{array}{l}\text { 3- } \\ \text { class }\end{array} & 8416.8 & 8378.7 & <.001 & \begin{array}{l}305 \\ (54.8 \%)\end{array} & \begin{array}{l}237 \\ (42.5 \%)\end{array} & \begin{array}{l}15 \\ (2.7 \%)\end{array} & \\ \begin{array}{l}4- \\ \text { class }\end{array} & 8375.4 & 8324.6 & .0002 & \begin{array}{l}302 \\ (54.2 \%)\end{array} & \begin{array}{l}208 \\ (37.3 \%)\end{array} & \begin{array}{l}29 \\ (5.2 \%)\end{array} & \begin{array}{l}18 \\ (3.2 \%)\end{array} & \\ \begin{array}{l}\text { 5- } \\ \text { class }\end{array} & 8345.9 & 8282.4 & .0813 & \begin{array}{l}262 \\ (47.0 \%)\end{array} & \begin{array}{l}199 \\ (35.7 \%)\end{array} & \begin{array}{l}48 \\ (8.6 \%)\end{array} & \begin{array}{l}31 \\ (5.6 \%)\end{array} & \begin{array}{l}(3.1 \%) \\ \hline\end{array}\end{array}$

Note. PD: Positive Deviant; BIC: Bayesian Information Criterion; ABIC: Adjusted Bayesian Information Criterion; BLRT: Bootstrapped Likelihood Ratio Test for k versus k-1 classes; class membership is sorted from largest to smallest.

Table 1

b. Concordance of group membership in suicidal attempts and mental and physical wellbeing $(n=557)$

2-class Class $1(n=502) \quad$ Class $2(n=55, P D)$

\begin{tabular}{|c|c|c|c|c|c|c|}
\hline & $\mathrm{n}$ & $\%$ & $\mathrm{n}$ & $\%$ & $x^{2}$ & $\mathrm{p}$ \\
\hline \multirow[t]{2}{*}{ Lifetime suicidal attempts } & 268 & $53.4 \%$ & 0 & $0.0 \%$ & 56.59 & .001 \\
\hline & $\begin{array}{l}\text { Mean } \\
\text { (SD) }\end{array}$ & Range & $\begin{array}{l}\text { Mean } \\
(\mathrm{SD})\end{array}$ & Range & $\mathrm{t}$ & $\mathrm{p}$ \\
\hline $\begin{array}{l}\text { SWEMWS mental } \\
\text { wellbeing }\end{array}$ & $17.5(2.7)$ & $\begin{array}{l}7.0- \\
30.7\end{array}$ & $22.4(2.4)$ & $17.4-29.3$ & -13.18 & $\begin{array}{l}<01 \\
.001\end{array}$ \\
\hline $\begin{array}{l}\text { EQ_VAS physical } \\
\text { wellbeing }\end{array}$ & $\begin{array}{l}46.3 \\
(20.2)\end{array}$ & $\begin{array}{l}0.0- \\
95.0\end{array}$ & $\begin{array}{l}79.1 \\
(10.9)\end{array}$ & $\begin{array}{l}40.0- \\
100.0\end{array}$ & -18.96 & $\begin{array}{l}<.001 \\
.001\end{array}$ \\
\hline
\end{tabular}




\subsection{Psychological and sociodemographic profiles of PD and non-PD cases}

Table 2 presents the differences between PD cases and non-PD cases on sociodemographic, mental and physical status, and emotion regulation and related factors. The PD cases had significantly higher proportions of individuals who received higher education $\left(47.3 \%\right.$ vs $\left.30.7 \%, \chi^{2}(1)=6.24, p=.012\right)$ and had higher perceived socioeconomic status ( 7.0 vs $5.6, t=7.24, d f=70.7, p<.001$ ) than the non-PD cases. Compared to the non-PD class, the PD cases had less severe suicidal thoughts $(8.1 \mathrm{vs} 19.9, t=$ $-10.31, d f=86.6, p<.001)$, less depression (10.0 vs $17.6, t=-9.65, d f=555, p<.001)$ and anxiety (7.6 vs $12.4, t=-6.52, d f=555, p<.001)$, more positive $(15.3$ vs $12.7, t=5.91, d f=555, p<.001)$ and less negative affect (13.1 vs $16.5, t=-7.52, d f=555, p<.001)$. 
Table 2

Characteristics of the participants based on class ( $\mathrm{n}=557)$

\begin{tabular}{|c|c|c|c|c|}
\hline & $\begin{array}{l}\text { PD cases } \\
(n=55)\end{array}$ & $\begin{array}{l}\text { Non-PD cases } \\
(n=502)\end{array}$ & & \\
\hline & $\mathrm{n}(\%) / \mathrm{M}(\mathrm{SD})$ & $\mathrm{n}(\%) / \mathrm{M}(\mathrm{SD})$ & $x^{2 / \mathrm{t}}$ & $\mathrm{p}$ \\
\hline Age & $22.2(2.6)$ & $21.9(2.3)$ & 0.99 & .320 \\
\hline Sex & & & 0.12 & .726 \\
\hline Male & $10(18.2 \%)$ & $82(16.3 \%)$ & & \\
\hline Female & $45(81.8 \%)$ & $420(83.7 \%)$ & & \\
\hline LGBTI status & & & 2.13 & .144 \\
\hline Yes & $23(41.8 \%)$ & $262(52.2 \%)$ & & \\
\hline No & $32(58.2 \%)$ & $240(47.8 \%)$ & & \\
\hline Location & & & 3.00 & .083 \\
\hline Rural/remote & $7(12.7 \%)$ & $115(22.9 \%)$ & & \\
\hline Metropolitan & $48(87.3 \%)$ & $387(77.1 \%)$ & & \\
\hline Living situation & & & 0.05 & .826 \\
\hline With family & $40(72.7 \%)$ & $358(71.3 \%)$ & & \\
\hline Others & $15(27.3 \%)$ & $144(28.7 \%)$ & & \\
\hline Relationship status & & & 1.39 & .238 \\
\hline Married, de facto, or in a relationship & $27(49.1 \%)$ & $205(40.8 \%)$ & & \\
\hline Others & $28(50.9 \%)$ & $297(59.2 \%)$ & & \\
\hline Education & & & 6.24 & .012 \\
\hline Diploma, bachelor, or above & $26(47.3 \%)$ & $154(30.7 \%)$ & & \\
\hline Others & $29(52.7 \%)$ & $348(69.3 \%)$ & & \\
\hline Physical health condition & & & 1.11 & .292 \\
\hline At least one long-term condition & $16(29.1 \%)$ & $182(36.3 \%)$ & & \\
\hline
\end{tabular}

Note. M: Mean; SD: standardized deviance; PD: positive deviant; LGBTI: lesbian, gay, bisexual, trans, and/or intersex; SIDAS: Suicidal Ideation Attributes Scale; PHQ-9: The Patient Health Questionnaire-9; GAD-7: General Anxiety Disorder-7; PANAS-SF: Positive and Negative Affect Schedule-Short Form; DERS-16: The Difficulties in Emotion Regulation Scale-16; CFS: Coping Flexibility Scale; CFS: Cognitive Flexibility Scale; RESE: Regulatory Emotional Self-efficacy Scale; Bold values indicate $p<$ 0.05 based on independent groups t-tests for continuous variables and $\chi 2$ tests for categorical variables, comparing PD and non-PD group. 


\begin{tabular}{|c|c|c|c|c|}
\hline & $\begin{array}{l}\text { PD cases } \\
(n=55)\end{array}$ & $\begin{array}{l}\text { Non-PD cases } \\
(n=502)\end{array}$ & & \\
\hline No long-term condition & $39(70.9 \%)$ & $320(63.7 \%)$ & & \\
\hline Mental health condition & & & 3.67 & .055 \\
\hline At least one diagnosis & $36(65.5 \%)$ & $387(77.1 \%)$ & & \\
\hline No diagnosed condition & $19(34.5 \%)$ & $115(22.9 \%)$ & & \\
\hline Current medication status & & & 3.57 & .059 \\
\hline Taking medication & $21(38.2 \%)$ & $259(51.6 \%)$ & & \\
\hline Not taking medication & $34(61.8 \%)$ & $243(48.4 \%)$ & & \\
\hline Socioeconomic status & $7.0(1.4)$ & $5.6(1.6)$ & 7.24 & $\begin{array}{l}<.001 \\
.001\end{array}$ \\
\hline SIDAS suicidal thoughts & $8.1(7.5)$ & $19.9(11.8)$ & -10.31 & $\begin{array}{l}<01 \\
.001\end{array}$ \\
\hline PHQ-9 depression & $10.0(5.1)$ & $17.6(5.6)$ & -9.65 & $\begin{array}{l}<.001 \\
.001\end{array}$ \\
\hline GAD-7 anxiety & $7.6(4.8)$ & $12.4(5.2)$ & -6.52 & $\begin{array}{l}< \\
.001\end{array}$ \\
\hline \multicolumn{5}{|l|}{ PANAS-SF affect } \\
\hline Positive affect & $15.3(3.4)$ & $12.7(3.1)$ & 5.91 & $\begin{array}{l}< \\
.001\end{array}$ \\
\hline Negative affect & $13.1(2.8)$ & $16.5(3.2)$ & -7.52 & $\begin{array}{l}<.001 \\
.001\end{array}$ \\
\hline DERS-16 emotion regulation & $46.8(12.1)$ & $57.6(12.4)$ & -6.14 & $\begin{array}{l}< \\
.001\end{array}$ \\
\hline CFS coping flexibility & $24.9(5.1)$ & $22.6(4.6)$ & 3.56 & $\begin{array}{l}< \\
.001\end{array}$ \\
\hline CFS cognitive flexibility & $54.4(6.8)$ & $46.8(7.4)$ & 7.26 & $\begin{array}{l}<.001 \\
.001\end{array}$ \\
\hline
\end{tabular}

Note. M: Mean; SD: standardized deviance; PD: positive deviant; LGBTI: lesbian, gay, bisexual, trans, and/or intersex; SIDAS: Suicidal Ideation Attributes Scale; PHQ-9: The Patient Health Questionnaire-9; GAD-7: General Anxiety Disorder-7; PANAS-SF: Positive and Negative Affect Schedule-Short Form; DERS-16: The Difficulties in Emotion Regulation Scale-16; CFS: Coping Flexibility Scale; CFS: Cognitive Flexibility Scale; RESE: Regulatory Emotional Self-efficacy Scale; Bold values indicate $p<$ 0.05 based on independent groups t-tests for continuous variables and $\chi 2$ tests for categorical variables, comparing PD and non-PD group. 


\begin{tabular}{|c|c|c|c|c|}
\hline & $\begin{array}{l}\text { PD cases } \\
(n=55)\end{array}$ & $\begin{array}{l}\text { Non-PD cases } \\
(n=502)\end{array}$ & & \\
\hline Expressing positive affect & $14.6(4.0)$ & $11.2(4.0)$ & 6.00 & $\begin{array}{l}<.001 \\
.001\end{array}$ \\
\hline Managing despondency distress & $9.4(3.3)$ & $7.8(3.3)$ & 3.51 & $\begin{array}{l}< \\
.001\end{array}$ \\
\hline Managing anger irritation & $10.3(3.6)$ & $8.8(3.4)$ & 3.08 & .002 \\
\hline \multicolumn{5}{|l|}{ Cognitive and behavioural responses } \\
\hline Digital technology & $2.9(0.7)$ & $3.1(0.6)$ & -2.38 & .018 \\
\hline Creative arts & $2.2(1.0)$ & $2.1(1.0)$ & 0.39 & .694 \\
\hline Self-harm and substance use & $1.9(0.6)$ & $2.5(0.7)$ & -6.31 & $\begin{array}{l}< \\
.001\end{array}$ \\
\hline Exercise & $2.1(0.9)$ & $1.8(0.8)$ & 3.36 & .001 \\
\hline Self-transcendence & $2.2(0.6)$ & $2.0(0.5)$ & 2.83 & .005 \\
\hline Self-care & $2.6(0.6)$ & $2.6(0.6)$ & 0.74 & .463 \\
\hline \multicolumn{5}{|c|}{$\begin{array}{l}\text { Note. M: Mean; SD: standardized deviance; PD: positive deviant; LGBTI: lesbian, gay, bisexual, trans, } \\
\text { and/or intersex; SID AS: Suicidal Ideation Attributes Scale; PHQ-9: The Patient Health Questionnaire-9; } \\
\text { GAD-7: General Anxiety Disorder-7; PANAS-SF: Positive and Negative Affect Schedule-Short Form; } \\
\text { DERS-16: The Difficulties in Emotion Regulation Scale-16; CFS: Coping Flexibility Scale; CFS: } \\
\text { Cognitive Flexibility Scale; RESE: Regulatory Emotional Self-efficacy Scale; Bold values indicate } p< \\
0.05 \text { based on independent groups t-tests for continuous variables and } \chi 2 \text { tests for categorical } \\
\text { variables, comparing PD and non-PD group. }\end{array}$} \\
\hline
\end{tabular}

The PD cases reported lower levels of difficulties in emotion regulation (46.8 vs $57.6, t=-6.14, d f=555, p$ $<.001$ ), higher coping flexibility (24.9 vs $22.6, t=3.56, d f=555, p<.001)$ and higher cognitive flexibility ( 54.4 vs $46.8, t=7.26, d f=555, p<.001$ ) than the non-PD cases. The PD cases also had higher levels of self-efficacy in expressing positive emotions (14.6 vs $11.2, t=6.00, d f=555, p<.001$ ), in managing despondency and distress (9.4 vs $7.8, t=3.51, d f=555, p<.001)$, and in managing anger and irritation (10.3 vs $8.8, t=3.08, d f=555, p=.002)$ than the non-PD cases. The PD cases were less likely to use digital technology (2.9 vs 3.1, $t=-2.38, d f=555, p=.018$ ) and less likely to use maladaptive coping behaviour including self-harm and substance use $(1.9$ vs $2.5, t=-6.31, d f=555, p<.001)$ as a response to emotional distress. They were more likely to engage in physical exercise (2.1 vs $1.8, t=3.36, d f=555, p$ $=.001$ ) and self-transcendence (2.2 vs $2.0, t=2.83, d f=555, p=.005$ ) than the non-PD cases. There was no significant difference between the PD and the non-PD cases on age, sex, sexual orientation, geographical location, living situation, relationship status, current medication status and likelihood of engaging in creative arts or self-care activities under emotional distress.

\subsection{Predictors of the PD class}

Predictors of the PD latent class are presented in Table 3. Having lower levels of emotion dysregulation ( $p$ $<.001)$, higher coping $(p=.001)$ or cognitive flexibility $(p<.001)$, great self-efficacy in expressing positive 
affect $(p<.001)$, reduced use of digital technology $(p=.019)$, less self-harm or substance use $(p<.001)$ as a response to emotional distress, more use of exercise $(p=.001)$ and self-transcendence $(p=.005)$ were associated with membership in the PD class. After adjustment for confounders (i.e., significant sociodemographic and mental health factors differentiating the PD and the non-PD classes), having higher cognitive flexibility $(p<.001)$, higher self-efficacy in expressing positive affect $(p=.013)$, reduced use of digital technology $(p=.005)$, and less self-harm and substance use $(p=.026)$ were significantly associated with PD class membership. 
Table 3

Logistic regression models of cognitive emotion regulation factors in predicting the PD latent class $(n=557)$

\begin{tabular}{|c|c|c|c|c|c|c|}
\hline & \multicolumn{2}{|c|}{ Unadjusted } & \multicolumn{4}{|c|}{ Adjusted } \\
\hline & OR & $95 \% \mathrm{Cl}$ & $\mathrm{p}$ & OR & $95 \% \mathrm{Cl}$ & $\mathrm{p}$ \\
\hline DERS-16 emotion regulation & 0.937 & $\begin{array}{l}0.916- \\
0.958\end{array}$ & $<.001$ & 0.996 & $\begin{array}{l}0.967- \\
1.026\end{array}$ & .783 \\
\hline CFS coping flexibility & 1.107 & $\begin{array}{l}1.045- \\
1.173\end{array}$ & .001 & 1.038 & $\begin{array}{l}0.966- \\
1.116\end{array}$ & .304 \\
\hline CFS cognitive flexibility & 1.164 & $\begin{array}{l}1.112- \\
1.219\end{array}$ & $<001$ & 1.114 & $\begin{array}{l}1.052- \\
1.179\end{array}$ & $<.001$ \\
\hline \multicolumn{7}{|l|}{$\begin{array}{l}\text { RESE regulatory emotional self- } \\
\text { efficacy }\end{array}$} \\
\hline Expressing positive affect & 1.219 & $\begin{array}{l}1.127- \\
1.318\end{array}$ & $\hat{.}_{001}$ & 1.119 & $\begin{array}{l}1.024- \\
1.222\end{array}$ & .013 \\
\hline Managing despondency distress & 1.042 & $\begin{array}{l}0.934- \\
1.162\end{array}$ & .464 & 0.944 & $\begin{array}{l}0.831- \\
1.073\end{array}$ & .378 \\
\hline Managing anger irritation & 1.064 & $\begin{array}{l}0.954- \\
1.187\end{array}$ & .267 & 1.047 & $\begin{array}{l}0.922- \\
1.189\end{array}$ & .475 \\
\hline \multicolumn{7}{|l|}{ Cognitive and behavioral responses } \\
\hline Digital technology & 0.604 & $\begin{array}{l}0.397- \\
0.919\end{array}$ & .019 & 0.474 & $\begin{array}{l}0.282- \\
0.798\end{array}$ & .005 \\
\hline Creative arts & 1.057 & $\begin{array}{l}0.803- \\
1.391\end{array}$ & .693 & 0.959 & $\begin{array}{l}0.702- \\
1.312\end{array}$ & .796 \\
\hline Self-harm and substance use & 0.234 & $\begin{array}{l}0.143- \\
0.383\end{array}$ & $\hat{.}_{.001}$ & 0.512 & $\begin{array}{l}0.284- \\
0.923\end{array}$ & .026 \\
\hline Exercise & 1.740 & $\begin{array}{l}1.248- \\
2.426\end{array}$ & .001 & 1.447 & $\begin{array}{l}0.969- \\
2.160\end{array}$ & .071 \\
\hline Self-transcendence & 2.010 & $\begin{array}{l}1.230- \\
3.285\end{array}$ & .005 & 1.220 & $\begin{array}{l}0.671- \\
2.218\end{array}$ & .514 \\
\hline Self-care & 1.197 & $\begin{array}{l}0.741- \\
1.932\end{array}$ & .462 & 0.934 & $\begin{array}{l}0.543- \\
1.606\end{array}$ & .806 \\
\hline \multicolumn{7}{|c|}{$\begin{array}{l}\text { Note. Bold values indicate } p<.05 \text {; PD: Positive Deviant; DERS-16: The Difficulties in Emotion } \\
\text { Regulation Scale-16; CFS: Coping Flexibility Scale; CFS: Cognitive Flexibility Scale; RESE: Regulatory } \\
\text { Emotional Self-efficacy Scale; the results were adjusted for age, sex, education, social economic } \\
\text { status, severity of suicidal thoughts, depression, and anxiety, and positive and negative affect. }\end{array}$} \\
\hline
\end{tabular}

\section{Discussion}


This study investigated suicide resilience using a positive deviance (PD) informed analytic approach. Using latent class analysis, a single PD class, who had high levels of wellbeing and an absence of suicide attempt, was identified. The PD class constitutes $10 \%$ of the total sample. The PD class was found to have less severe suicidal thoughts, greater positive affect, and less negative affect than the non-PD class as expected. There were few sociodemographic factors that differentiated the PD group from the non-PD group, other than significantly greater rates of tertiary educational attainment and higher socioeconomic status.

Greater cognitive flexibility, higher self-efficacy in expressing positive affect, and adaptive emotion regulation strategies, including reduced use of digital technology and reduced self-harm and substance use were significantly associated with membership of the PD class after adjusting for sociodemographic and mental health factors. Associations between PD class and emotion dysregulation, coping flexibility, more use of exercise and self-transcendence were significant in bivariate analysis but not significant in adjusted models. These findings indicate a number of potentially important foci for interventions to support individuals with current suicidal experience.

Cognitive flexibility but not coping flexibility appears to be strongly associated with positive deviance. Approximately one standard deviation increase in cognitive flexibility was associated with double odds of positive deviance. This finding is in large consistent with the literature indicating that psychotherapies (e.g., cognitive behavioural therapy) that appear to increase cognitive flexibility ${ }^{58}$ are effective for reducing suicidal thoughts and behaviour ${ }^{59,60}$. While cognitive reframing is a key focus of $C B T$, the current findings suggest that providing an explicit focus on cognitive flexibility for people with suicidal thoughts may increase their resilience to suicidal behaviour. Cognitive flexibility may be a potential mechanism as to how cognitive restructuring impacts suicidal thoughts and behaviour but this explanation warrants further experimental investigation. We found no significant relationship between coping flexibility and positive deviance after adjusting for current mental health status and sociodemographic characteristics. This finding is consistent with previous findings ${ }^{38}$ indicating the moderating effects of coping flexibility on the relationship between depressive symptoms and suicide risks. Future studies may investigate the interaction between coping flexibility and mental health symptoms in individuals with suicidal thoughts or behaviour in a larger sample size.

Our study indicates a significant relationship between regulatory emotional self-efficacy and the PD membership, specifically through greater expression of positive affect. This finding echoes the increasing literature suggesting the important role of regulatory emotional self-efficacy in suicide prevention ${ }^{61,62}$. A recent study indicates significant mediating effects of regulatory emotional self-efficacy, but not acquired capability for suicide, on the relationships between nonsuicidal self-injury frequency and lifetime suicide attempts in both community-based and clinic samples ${ }^{40}$. Our findings extend this knowledge and suggest that regulatory emotional self-efficacy in expressing positive affect, but not managing despondency distress or anger irritation may play a critical role in the transition to suicidal attempts. Further study to unveil the factors that differentiate the impacts of domains of regulatory emotional self- 
efficacy, for example, social support, self-esteem, or personality, may help in understanding the mechanisms underlying this association.

The findings around the use of specific coping behaviour also points to the focus areas where health promotion and clinical interventions may benefit young people at risk of suicide. Young adults in the PD class were less likely to use digital technology to cope with emotional distress. While recent reviews indicate that minimum impact of digital technology use on mental health symptoms ${ }^{63,64}$, our findings suggest that using digital technology (e.g., TV, online videos, social media) as a distraction to emotional distress can be a maladaptive coping strategy amongst young adults at risk of suicide. This is consistent with previous research reporting that use of digital technology is not effective for coping with distress ${ }^{65}$ and associated with lower levels of psychological wellbeing ${ }^{66}$.

Those in the PD class were also less likely to use substances or self-harm to cope with emotional distress, echoing previous findings on the roles of self-harm ${ }^{67}$ and substance use ${ }^{68}$ in suicidal behaviour and a recent cohort study focusing on the transition from suicidal thoughts to behaviour amongst adolescents ${ }^{69}$. Although physical activity and practice of self-transcendence (e.g., prayer, mindfulness or meditation) were significantly associated with the PD class in the bivariate associations, they became non-significant in the adjusted models. This finding is in large consistent with the qualitative research in suicide prevention ${ }^{70}$, suggesting the difficulties in practicing mindfulness and exercise during a crisis, particularly when fatigue is present. Nevertheless, physical activity has also been shown to improve cognitive flexibility in adults ${ }^{58}$, suggesting that behavioural activation and other health behaviour change approaches to increase physical activity may also aid in individuals' coping with suicidal experience, outside of a crisis.

Interestingly, emotion dysregulation was not significant in the adjusted model. Previous reviews have suggested that dialectical behaviour therapy (DBT), which focuses on emotion regulation, is associated with only modest reductions in suicidal thoughts ${ }^{60}$, although effects tend to be larger among people with personality disorders ${ }^{71}$. Our findings are consistent with previous findings indicating: (1) that the relationship between emotion regulation and wellbeing is complex and largely explained by mental health status ${ }^{72}$, (2) that emotion regulation may only impact wellbeing for certain subgroups of the population ${ }^{73}$, and (3) that observed effects are only related to specific emotion regulation strategies ${ }^{73}$. In combination, the findings suggest that a sole focus on emotion regulation is unlikely to be an optimal universal approach for suicide prevention interventions.

This study was the first to apply a positive deviance framework to identifying resilience to suicidal behaviour, using a large non-clinical sample of young adults with recent suicidal thought. We found $10 \%$ of the surveyed population met the criteria of PD. This number aligns with the previous estimate that PDs typically account for $0 \%$ to $10 \%$ of a population ${ }^{74}$. There were a few meaningful differences between the PD and the non-PD classes, which provides a potential practical approach to revisit the evidence in suicide prevention research. 
Some limitations to this study need to be acknowledged. The direction and causation of significant associations could not be established due to the cross-sectional nature of the study. Nevertheless, the findings of this research suggest potential novel targets for further clinical, experimental and health promotion research. Examining longitudinal outcomes for people identified as positive deviants, including suicidal thoughts, suicide attempts and wellbeing, would also be an important extension of this research. Due to the epidemiological approach and distal assessment methodology adopted in this research, all of the outcomes were based on self-report, without clinician verification of mental health outcomes. Although established, validated measures were used, examination of positive deviance in a clinical context may reveal other factors that support resilience to suicidal behaviour. There may have been factors associated with positive deviance that we did not measure, which may be worthy of future investigation. Such factors may include personality, social connectedness and hopelessness. Finally, the recruitment strategy is unlikely to result in a sample that was representative of the population of interest. We chose online recruitment because young adults have high use of social media and low rates of service use. Nevertheless, variability in social media use and trust in advertising may have influenced the composition of the sample.

\section{Conclusions}

This study demonstrated a methodology to understand suicidal resilience by identifying positive deviants, who comprised approximately $10 \%$ of a non-clinical sample with suicidal thoughts. We found that greater cognitive flexibility, greater self-efficacy in expression of positive affect, reduced use of digital technology, and less self-harm and substance use for coping were associated with greater resilience to suicidal behaviour. These findings suggest that specific emphasis on cognitive flexibility, regulatory emotional self-efficacy, and avoidance of maladaptive coping in therapeutic and health promotion interventions may be important for increasing wellbeing and reducing suicidal behaviour among young adults. A greater focus on strengths-based approaches to suicide prevention may benefit young adults at risk of suicide.

\section{Declarations}

\section{Author contributions}

All authors have made significant contributions to the manuscript. JH designed the study with support from $\mathrm{HC}$ and PJB. JH and IW implemented the study. JH and PJB planned the statistical analysis, and JH analyzed the data. All authors contributed to the writing and edits of the manuscript.

\section{Acknowledgements}

JH is supported by the Commonwealth Suicide Prevention Research Fund Post-Doctoral Fellowship. PJB and HC are supported by NHMRC Fellowships 1158707 and 1155614, respectively.

\section{Competing interests}


The authors declare no competing interests.

\section{Data availability}

The data that support the findings of this study are available on request from the corresponding author, $\mathrm{JH}$. The data are not publicly available due to ethics restrictions on the privacy of research participants.

\section{References}

1. Australian Institute of Health and Welfare. Deaths in Australia. Cat. no. PHE 229., <https://www.aihw.gov.au/reports/life-expectancy-death/deaths-in-australia> (2021).

2. Gallagher, M. L. \& Miller, A. B. Suicidal Thoughts and Behavior in Children and Adolescents: An Ecological Model of Resilience. Adolesc Res Rev 3, 123-154, doi:10.1007/s40894-017-0066-Z (2018).

3. Shahram, S. Z. et al. Promoting "Zest for Life": A Systematic Literature Review of Resiliency Factors to Prevent Youth Suicide. J Res Adolesc 31, 4-24, doi:10.1111/jora.12588 (2021).

4. Reifels, L. et al. Research priorities in suicide prevention: Final report. (The University of Melbourne, Melbourne, 2017).

5. Sher, L. Resilience as a focus of suicide research and prevention. Acta Psychiatrica Scandinavica 140, 169-180, doi:https://doi.org/10.1111/acps.13059 (2019).

6. Ivbijaro, G. et al. Preventing suicide, promoting resilience: Is this achievable from a global perspective? Asia-Pacific Psychiatry 11, e12371, doi:10.1111/appy.12371 (2019).

7. Kessler, R. C., Bossarte, R. M., Luedtke, A., Zaslavsky, A. M. \& Zubizarreta, J. R. Suicide prediction models: A critical review of recent research with recommendations for the way forward. Mol Psychiatry 25, 168-179, doi:10.1038/s41380-019-0531-0 (2020).

8. Johnson, J., Wood, A. M., Gooding, P., Taylor, P. J. \& Tarrier, N. Resilience to suicidality: the buffering hypothesis. Clin Psychol Rev 31, 563-591, doi:10.1016/j.cpr.2010.12.007 (2011).

9. Sher, L. Primary and secondary resilience to stress-related disorders and suicidal behavior. Aust N Z J Psychiatry 54, 108, doi:10.1177/0004867419876698 (2020).

10. Fergusson, D. M., Beautrais, A. L. \& Horwood, L. J. Vulnerability and resiliency to suicidal behaviours in young people. Psychol Med 33, 61-73, doi:10.1017/s0033291702006748 (2003).

11. Kessler, R. C., Borges, G. \& Walters, E. E. Prevalence of and risk factors for lifetime suicide attempts in the National Comorbidity Survey. Arch Gen Psychiatry 56, 617-626, doi:10.1001/archpsyc.56.7.617 (1999).

12. Collins, K. R. L., Stritzke, W. G. K., Page, A. C., Brown, J. D. \& Wylde, T. J. Mind full of life: Does mindfulness confer resilience to suicide by increasing zest for life? J Affect Disord 226, 100-107, doi:10.1016/j.jad.2017.09.043 (2018).

13. Siegmann, P. et al. Resilience to suicide ideation: A cross-cultural test of the buffering hypothesis. Clin Psychol Psychother 25, e1-e9, doi:10.1002/cpp.2118 (2018). 
14. Luthar, S. S. Annotation: methodological and conceptual issues in research on childhood resilience. J Child Psychol Psychiatry 34, 441-453, doi:10.1111/j.1469-7610.1993.tb01030.x (1993).

15. Herington, M. J. \& van de Fliert, E. Positive Deviance in Theory and Practice: A Conceptual Review. Deviant Behav 39, 664-678, doi:10.1080/01639625.2017.1286194 (2018).

16. Sternin, M., Sternin, J. \& Marsh, D. Designing a Community-Based Nutrition Program Using the Hearth Model and the Positive Deviance Approach - A Field Guide. (Save the Children, 1998).

17. Zeitlin, M. F., Ghassemi, H., Mansour, M., United Nations University \& Joint WHO/UNICEF Nutrition Support Programme. (United Nations University Press, Tokyo, 1990).

18. Pascale, R., Sternin, J. \& Sternin, M. The Power Of Positive Deviance. (Harvard Business Review Press, 2010).

19. Bisits Bullen, P. A. The positive deviance/hearth approach to reducing child malnutrition: systematic review. TM \& IH 16, 1354-1366, doi:10.1111/j.1365-3156.2011.02839.x (2011).

20. Babalola, S., Awasum, D. \& Quenum-Renaud, B. The correlates of safe sex practices among Rwandan youth: a positive deviance approach. Afr J AIDS Res 1, 11-21, doi:10.2989/16085906.2002.9626540 (2002).

21. Banerjee, E. S. et al. Determinants of Successful Weight Loss in Low-Income African American Women: A Positive Deviance Analysis. J Prim Care Community Health 9, 2150132718792136, doi:10.1177/2150132718792136 (2018).

22. The Positive Deviance Initiative. Basic Field Guide to the Positive Deviance Approach. (Boston, MA, 2010).

23. Berman, A. L. Risk Factors Proximate to Suicide and Suicide Risk Assessment in the Context of Denied Suicide Ideation. Suicide Life Threat Behav 48, 340-352, doi:10.1111/sltb.12351 (2018).

24. World Health Organization. Suicide, <https://www.who.int/news-room/fact-sheets/detail/suicide> (2021).

25. Law, K. C., Khazem, L. R. \& Anestis, M. D. The role of emotion dysregulation in suicide as considered through the ideation to action framework. Curr Opin Psychol 3, 30-35, doi:https://doi.org/10.1016/j.copsyc.2015.01.014 (2015).

26. Hatkevich, C., Penner, F. \& Sharp, C. Difficulties in emotion regulation and suicide ideation and attempt in adolescent inpatients. Psychiatry Res 271, 230-238, doi:10.1016/j.psychres.2018.11.038 (2019).

27. Brausch, A. M. \& Woods, S. E. Emotion Regulation Deficits and Nonsuicidal Self-Injury Prospectively Predict Suicide Ideation in Adolescents. Suicide Life Threat Behav 49, 868-880, doi:10.1111/sltb.12478 (2019).

28. Neacsiu, A. D., Fang, C. M., Rodriguez, M. \& Rosenthal, M. Z. Suicidal Behavior and Problems with Emotion Regulation. Suicide Life Threat Behav 48, 52-74, doi:10.1111/sltb.12335 (2018).

29. Cong, C. W., Ling, W. S. \& Fitriana, M. Family functioning, coping strategy, and suicidal ideation among adolescents. J Child Adolesc Ment Health 32, 131-140, 
doi:10.2989/17280583.2020.1848852 (2020).

30. Weiss, N. H., Sullivan, T. P. \& Tull, M. T. Explicating the role of emotion dysregulation in risky behaviors: A review and synthesis of the literature with directions for future research and clinical practice. Curr Opin Psychol 3, 22-29, doi:10.1016/j.copsyc.2015.01.013 (2015).

31. Wolff, J. C. et al. Emotion dysregulation and non-suicidal self-injury: A systematic review and metaanalysis. Eur Psychiatry 59, 25-36, doi:10.1016/j.eurpsy.2019.03.004 (2019).

32. Ghorbani, F., Khosravani, V., Sharifı Bastan, F. \& Jamaati Ardakani, R. The alexithymia, emotion regulation, emotion regulation difficulties, positive and negative affects, and suicidal risk in alcoholdependent outpatients. Psychiatry Res 252, 223-230, doi:10.1016/j.psychres.2017.03.005 (2017).

33. Van Orden, K. A. et al. The interpersonal theory of suicide. Psychol Rev 117, 575-600, doi:https://doi.org/10.1037/a0018697 (2010).

34. Chapman, A. L., Gratz, K. L. \& Brown, M. Z. Solving the puzzle of deliberate self-harm: The experiential avoidance model. Behav Res Ther 44, 371-394, doi:https://doi.org/10.1016/j.brat.2005.03.005 (2006).

35. Anestis, M. D., Bagge, C. L., Tull, M. T. \& Joiner, T. E. Clarifying the role of emotion dysregulation in the interpersonal-psychological theory of suicidal behavior in an undergraduate sample. J Psychiatr Res 45, 603-611, doi:10.1016/j.jpsychires.2010.10.013 (2011).

36. Anestis, M. D., Knorr, A. C., Tull, M. T., Lavender, J. M. \& Gratz, K. L. The importance of high distress tolerance in the relationship between nonsuicidal self-injury and suicide potential. Suicide Life Threat Behav 43, 663-675, doi:10.1111/sltb.12048 (2013).

37. Heffer, T. \& Willoughby, T. A count of coping strategies: A longitudinal study investigating an alternative method to understanding coping and adjustment. PLoS One 12, e0186057, doi:10.1371/journal.pone.0186057 (2017).

38. Kato, T. Moderation Effects of Coping Flexibility on the Association Between Depressive Symptoms and Suicidal Risk. Crisis, 1-6, doi:10.1027/0227-5910/a000800 (2021).

39. Mai, Y. et al. Impulsiveness and suicide in male offenders: Examining the buffer roles of regulatory emotional self-efficacy and flourishing. Psychol Psychother 94, 289-306, doi:10.1111/papt.12312 (2021).

40. Gratz, K. L., Spitzen, T. L. \& Tull, M. Expanding our understanding of the relationship between nonsuicidal self-injury and suicide attempts: The roles of emotion regulation self-efficacy and the acquired capability for suicide. J Clin Psychol 76, 1653-1667 (2020).

41. Adler, N. E., Epel, E. S., Castellazzo, G. \& Ickovics, J. R. Relationship of subjective and objective social status with psychological and physiological functioning: preliminary data in healthy white women. Health Psychol 19, 586-592, doi:10.1037//0278-6133.19.6.586 (2000).

42. Fat, L. N., Scholes, S., Boniface, S., Mindell, J. \& Stewart-Brown, S. Evaluating and establishing national norms for mental wellbeing using the short Warwick-Edinburgh Mental Well-being Scale (SWEMWBS): findings from the Health Survey for England. Qual. Life Res. 26, 1129-1144, doi:10.1007/s11136-016-1454-8 (2017). 
43. EuroQol Group. EuroQol-a new facility for the measurement of health-related quality of life. Health Policy 16, 199-208, doi:10.1016/0168-8510(90)90421-9 (1990).

44. van Spijker, B. A. et al. The suicidal ideation attributes scale (SIDAS): Community-based validation study of a new scale for the measurement of suicidal ideation. Suicide Life Threat Behav 44, 408419, doi:10.1111/sltb.12084 (2014).

45. van Spijker, B. A. et al. Effectiveness of a web-based self-help program for suicidal thinking in an Australian community sample: Randomized controlled trial. J Med Internet Res 20, e15, doi:10.2196/jmir.8595 (2018).

46. Kroenke, K., Spitzer, R. L. \& Williams, J. B. The PHQ-9: validity of a brief depression severity measure. J Gen Intern Med 16, 606-613, doi:10.1046/j.1525-1497.2001.016009606.x (2001).

47. Spitzer, R. L., Kroenke, K., Williams, J. B. W. \& Löwe, B. A Brief Measure for Assessing Generalized Anxiety Disorder: The GAD-7. Arch Intern Med 166, 1092-1097, doi:10.1001/archinte.166.10.1092 (2006).

48. Thompson, E. R. Development and Validation of an Internationally Reliable Short-Form of the Positive and Negative Affect Schedule (PANAS). J Cross Cult Psychol 38, 227-242, doi:10.1177/0022022106297301 (2007).

49. Bjureberg, J. et al. Development and Validation of a Brief Version of the Difficulties in Emotion Regulation Scale: The DERS-16. J Psychopathol Behav Assess 38, 284-296, doi:10.1007/s10862015-9514-x (2016).

50. Kato, T. Development of the Coping Flexibility Scale: evidence for the coping flexibility hypothesis. J Couns Psychol 59, 262-273, doi:10.1037/a0027770 (2012).

51. Martin, M. M. \& Rubin, R. B. A New Measure of Cognitive Flexibility. Psychol Rep 76, 623-626, doi:10.2466/pro.1995.76.2.623 (1995).

52. Caprara, G. V. et al. Assessing regulatory emotional self-efficacy in three countries. Psychol Assess 20, 227-237, doi:10.1037/1040-3590.20.3.227 (2008).

53. Skinner, E. A. \& Zimmer-Gembeck, M. J. The Development of Coping. Annu Rev Psychol 58, 119-144, doi:10.1146/annurev.psych.58.110405.085705 (2007).

54. Han, J. et al. A Mobile Health Intervention (LifeBuoy App) to Help Young People Manage Suicidal Thoughts: Protocol for a Mixed-Methods Randomized Controlled Trial. JMIR Res Protoc 9, e23655e23655, doi:10.2196/23655 (2020).

55. Collins, L. \& Lanza, S. Latent Class and Latent Transition Analysis: With Applications in the Social, Behavioral, and Health Sciences. (John Wiley and Sons Inc., 2010).

56. Wong, Q. J. J. et al. Identifying subgroups within a sample of adults with a suicide attempt history using the Interpersonal Psychological Theory of Suicide. Psychiatry Res 293, 113406, doi:10.1016/j.psychres.2020.113406 (2020).

57. Nylund, K. L., Asparouhov, T. \& Muthén, B. O. Deciding on the Number of Classes in Latent Class Analysis and Growth Mixture Modeling: A Monte Carlo Simulation Study. Struct Equ 14, 535-569, doi:10.1080/10705510701575396 (2007).

Page 20/22 
58. Murata, T. et al. Alterations of mental defeat and cognitive flexibility during cognitive behavioral therapy in patients with major depressive disorder: a single-arm pilot study. BMC Res. Notes 12, 723, doi:10.1186/s13104-019-4758-2 (2019).

59. Zalsman, G. et al. Suicide prevention strategies revisited: 10-year systematic review. Lancet Psychiatry 3, 646-659, doi:10.1016/S2215-0366(16)30030-X (2016).

60. D'Anci, K. E., Uhl, S., Giradi, G. \& Martin, C. Treatments for the Prevention and Management of Suicide. Ann Intern Med 171, 334-342, doi:10.7326/M19-0869 (2019).

61. Valois, R. F., Zullig, K. J. \& Hunter, A. A. Association Between Adolescent Suicide Ideation, Suicide Attempts and Emotional Self-Efficacy. J Child Fam Stud 24, 237-248, doi:10.1007/s10826-0139829-8 (2015).

62. Liu, S., You, J., Ying, J., Li, X. \& Shi, Q. Emotion reactivity, nonsuicidal self-injury, and regulatory emotional self-efficacy: A moderated mediation model of suicide ideation. J Affect Disord 266, 8289, doi:10.1016/j.jad.2020.01.083 (2020).

63. Tang, S., Werner-Seidler, A., Torok, M., Mackinnon, A. J. \& Christensen, H. The relationship between screen time and mental health in young people: A systematic review of longitudinal studies. Clin Psychol Rev 86, 102021, doi:https://doi.org/10.1016/j.cpr.2021.102021 (2021).

64. Scott, H. \& Woods, H. C. Understanding Links Between Social Media Use, Sleep and Mental Health: Recent Progress and Current Challenges. Curr Sleep Med Rep 5, 141-149, doi:10.1007/s40675-01900148-9 (2019).

65. Duvenage, M. et al. Technology can sting when reality bites: Adolescents' frequent online coping is ineffective with momentary stress. Comput Hum Behav 102, 248-259, doi:https://doi.org/10.1016/j.chb.2019.08.024 (2020).

66. Büchi, M., Festic, N. \& Latzer, M. Digital Overuse and Subjective Well-Being in a Digitized Society. Soc. Media Soc 5, 2056305119886031, doi:10.1177/2056305119886031 (2019).

67. Owens, D., Horrocks, J. \& House, A. Fatal and non-fatal repetition of self-harm: Systematic review. $\mathrm{Br}$ J Psychiatry 181, 193-199, doi:10.1192/bjp.181.3.193 (2002).

68. Rioux, C. et al. Substance use disorders and suicidality in youth: A systematic review and metaanalysis with a focus on the direction of the association. PLoS One 16, e0255799, doi:10.1371/journal.pone.0255799 (2021).

69. Mars, B. et al. Predictors of future suicide attempt among adolescents with suicidal thoughts or nonsuicidal self-harm: a population-based birth cohort study. Lancet Psychiatry 6, 327-337, doi:10.1016/s2215-0366(19)30030-6 (2019).

70. Chesin, M. S. et al. Perceptions of adjunctive mindfulness-based cognitive therapy to prevent suicidal behavior among high suicide-risk outpatient participants. Crisis 39, 451-460, doi:http://dx.doi.org/10.1027/0227-5910/a000519 (2018).

71. Panos, P. T., Jackson, J. W., Hasan, O. \& Panos, A. Meta-Analysis and Systematic Review Assessing the Efficacy of Dialectical Behavior Therapy (DBT). Res Soc Work Pract 24, 213-223, doi:10.1177/1049731513503047 (2013). 
72. Chervonsky, E. \& Hunt, C. Emotion regulation, mental health, and social wellbeing in a young adolescent sample: A concurrent and longitudinal investigation. Emotion 19, 270-282, doi:10.1037/emo0000432 (2019).

73. Brewer, S. K., Zahniser, E. \& Conley, C. S. Longitudinal impacts of emotion regulation on emerging adults: Variable- and person-centered approaches. J Appl Dev Psychol 47, 1-12, doi:https://doi.org/10.1016/j.appdev.2016.09.002 (2016).

74. Marsh, D. R., Schroeder, D. G., Dearden, K. A., Sternin, J. \& Sternin, M. The power of positive deviance. BMJ 329, 1177-1179, doi:10.1136/bmj.329.7475.1177 (2004). 